\title{
First do no harm: overdiagnosis in Pediatrics
}

\author{
Virginia A. Moyer, $M D, M P H^{a}$
}

\begin{abstract}
Many errors can be made in diagnosis: underdiagnosis, misdiagnosis, and overdiagnosis. While underdiagnosis and misdiagnosis are clear errors, in overdiagnosis, a true abnormality is discovered, but detection does not benefit the patient. Harm occurs when patients are further evaluated and treated unnecessarily as a result of making a diagnosis that would never have affected the patient if the diagnosis had not been made. Several phenomena point to potential overdiagnosis: when delayed or missed diagnoses do not result in harm; when there is increased detection of a disease, but no change in the outcome; and when randomized trials show no benefit from the diagnosis. Some might say that there is always benefit in knowing, but the adverse effects of overdiagnosis are well documented. We will need to educate ourselves and our colleagues about the potential for harm from overdiagnosis, and learn how to balance the potential benefit of a diagnosis against the risk of overdiagnosis.

Key words: overdiagnosis, diagnostic errors, unnecessary procedures.
\end{abstract}

http: / / dx.doi.org/10.5546/ aap.2018.eng.426

To cite: Moyer VA. First do no harm: overdiagnosis in Pediatrics. Arch Argent Pediatr 2018;116(6):426-429.

Overdiagnosis is a new concept, especially in pediatrics, which we know from the current literature: fewer than $10 \%$ of the articles in Medline about overdiagnosis relate to children. What is "overdiagnosis" and why is it important for children? Many errors can be made in diagnosis: underdiagnosis, misdiagnosis, and overdiagnosis. While underdiagnosis (missing something that is present) and misdiagnosis (making the wrong diagnosis) are clear errors, overdiagnosis is different. In overdiagnosis, a true abnormality is discovered, but detection does not benefit the patient. This seems counterintuitive: how can making a correct diagnosis not benefit the patient or even result in harm? Harm occurs when patients are further evaluated and treated unnecessarily as a result of making a diagnosis that would never have affected the patient if the diagnosis had not been made. Some examples include neuroblastoma diagnosed by screening in young infants, asymptomatic bacteruria treated as infection, mild vesico-ureteral reflux, and nondisplaced linear skull fractures.

Recently, it has been recognized that by "medicalizing" variants of normal, we may be promoting overtreatment of children who are simply on one end of the normal distribution of symptoms or signs. For example, ADHD is more often diagnosed in the youngest children in a grade level. There is no reason to believe that the true incidence of ADHD is different among younger than slightly older children, the younger children simplydisplay more immature behavior. ${ }^{1}$ Similarly, when ordinary spitting up, which affects $50 \%$ of otherwise healthy infants, is labeled as "gastroesophageal reflux disease" rather than simply gastroesophageal reflux (or even just "spitting up"), their parents are more likely to ask for medication. ${ }^{2}$

Overdiagnosis can also occur because some new tests are too good. As test sensitivity increases, milder and milder levels of a condition will be uncovered, for which treatment is less and less likely to be useful. Most mild vesico-ureteral reflux resolves spontaneously, and no interventions have been shown to reduce renal scarring or insufficiency. ${ }^{3}$ Similarly, ultrasound screening of the hips in the first weeks of life in infants without risk factors reveals a high number with possible abnormality -but $90 \%$ of these resolve without treatment within weeks to months. ${ }^{4}$ CT scan may reveal isolated linear skull fracture in minor head injury- 
these have uniformly good outcome, but children are frequently subjected to hospitalization and repeat CT scanning. ${ }^{5}$

\section{How can we recognize when overdiagnosis is occurring?}

Several phenomena point to potential overdiagnosis: when delayed or missed diagnoses do not result in harm; when there is increased detection of a disease, but no change in the outcome; and when randomized trials show no benefit from the diagnosis. One current example of overdiagnosis in pediatric patients is the detection of mild hypoxemia in bronchiolitis, which leads to overuse of supplemental oxygen, increased hospitalization for bronchiolitis, and increased length of hospital stay. ${ }^{6}$ To test whether mild hypoxemia is overdiagnosed in young children with bronchiolitis, the occurrence of desaturations was evaluated in children with mild bronchiolitis not requiring hospitalization: 118 infants were discharged from the ED with an $\mathrm{O}_{2}$ saturation monitor that had no alarm function and no display. All of these infants did well, with no increase in unscheduled emergency visits or hospital admission. However, on the monitor recordings, $64 \%$ of the infants had $\mathrm{O}_{2}$ sat $<90 \%$ for at least one minute, and $25 \%$ had recorded $\mathrm{O}_{2}$ saturations down to $70 \%$. This "diagnosis" of mild hypoxemia was not associated with any adverse outcomes. $^{7}$

Overdiagnosis should be suspected when there is increased detection of a disease over time, but no change in outcome. As the use of pulse oximetry has increased, admissions for bronchiolitis have tripled in the US, however, mortality rates from bronchiolitis have not changed. This suggests that children with very mild bronchiolitis are being diagnosed with hypoxia, and treated as if they were more severe cases of bronchiolitis. ${ }^{8,9}$

Finally, the hypothesis that overdiagnosis has occurred can be empirically tested. Two randomized trials of screening for hypoxemia have demonstrated that increasing the perception of hypoxemia results in increased medical intervention, even when the child is doing well. ${ }^{10,11}$

These trials randomized children with bronchiolitis to different displays of levels of hypoxia. One trial used intermittent vs continuous display and the other trial showed skewed saturation values on the display so that infants with the same actual oxygen saturation had different displays. In both cases, the higher the displayed oxygen saturation, the lower the rate of hospitalization and / or shorter the hospital stay. When fewer children were diagnosed with hypoxia, they received the less medical intervention, with no change in outcomes.

\section{Why is overdiagnosis a problem?}

Some might say that there is always benefit in knowing, but the adverse effects of overdiagnosis are well documented. One is the direct risk from testing itself. Head CT for mild head trauma involves significant exposure to radiation, with a predictable increase in lifetime cancer risk. If a child has several CTs in follow up of a linear skull fracture, his lifetime brain cancer risk is substantially increased. ${ }^{12}$ Another risk is labeling the child as diseased. The "Vulnerable Child Syndrome", described more than 50 years ago related to innocent heart murmurs, resulted in perfectly healthy children having their physical activity restricted, even years later. ${ }^{13}$ Disease labels also increase belief in medication, even when it is known that medication is likely not effective. Only harm can result from treatment aimed at any diagnosis for which treatment is not needed. Immediate surgical management of infantile neuroblastoma has been associated with a $2 \%$ mortality risk, ${ }^{14}$ while observation as a primary strategy has been associated with $100 \%$ survival. ${ }^{15}$ Unnecessary antibiotic use, as in mild vesico-ureteral reflux, contributes to antibiotic resistance, harming both the individual child and other children in the population. ${ }^{16}$ Finally, overdiagnosis and consequent unnecessary management contributes to cost, and contributes to waste in the system - the very definition of low value care.

\section{Why do we over diagnose?}

For most physicians, even in primary care, uncertainty is uncomfortable: we are more afraid of missing something and looking foolish than we are of making a false diagnosis or of overdiagnosis. Ordering fewer tests can be time consuming, as it might require more "hands-on" time for added clinical follow up and more time for discussion with the family. "The identification and correction of physiologic abnormalities is ingrained in medical culture". ${ }^{17}$ Physicians may perceive that patients (or parents) want additional testing, so that physicians and patients together are "co-conspirators in a behavioral system that often sacrifices safety for action". ${ }^{18}$ In fact, when overdiagnosis results in harm, then 
doing something "just to be safe" may actually undermine our efforts in patient safety. ${ }^{6}$

\section{How can we do better?}

We live by the maxim of Primum non nocere, that is, "First do no harm". We will need to educate ourselves and our colleagues about the potential for harm from overdiagnosis, and learn how to balance the potential benefit of a diagnosis against the risk of overdiagnosis. The "Choosing Wisely" campaign in the US has listed at least 5, and often more, tests and treatments that are generally not needed for each of a wide variety of specialties and populations. ${ }^{19}$ For pediatrics alone, 154 items relevant to children are already listed, including the use of CT scans in several situations, and treatment of several diagnoses for which treatment does not provide net benefit.

We should ask the key question: Will the patient be better off for having the test? Current quality measures related to diagnostic testing often focus on underuse of diagnostic or screening tests; we can design quality measures to address common sources of overdiagnosis, and develop measures based on implementation of guidelines. We can also develop measures based on value: what does each test or diagnosis add to the health of the patient? Finally, we can include errors of commission in adverse event reviews, and ask whether the test or intervention that caused harm was warranted in the first place. As always, research is needed to expand our knowledge about overdiagnosis. Researchers should identify and study potentially overdiagnosed conditions, critically evaluate accepted practices, and focus on test value, not accuracy.

Wise people saw this coming many years ago. Richard Behrman, past editor of the Nelson Textbook of Pediatrics, said this in 1996: "Misperceptions of disease prevalence and therapeutic effectiveness can promote a cycle of increasing medical intervention, despite the best intentions of all parties. The cycle usually begins with some form of increased testing that lowers the threshold for detecting diseasesuch as technical improvement in imaging, more frequent testing, or closer scrutiny of the images-which immediately leads to a higher diagnostic yield of the disease and a spectrum of milder disease, which then may be unnecessarily treated with pseudosuccess" ${ }^{20}$

\section{Further reading and resources}

- Overdiagnosed: Making People Sick in the Pursuit of Health 2011 Gilbert Welch, Lisa Schwartz, Steven
Woloshin.

- Selling Sickness 2009 Ray Moynihan and Alan Cassels.

- Preventing Overdiagnosis

Annual Conference (Copenhagen in 2018). www.preventingoverdiagnosis.net

\section{REFERENCES}

1. Morrow RL, Garland EJ, Wright JM, et al. Influence of relative age on diagnosis and treatment of attention-deficit/ hyperactivity disorder in children. CMAJ 2012; 184(7):755-62.

2. Carey WB. The hazards of medicalizing variants of normal. Pediatrics 2013; 131(5):991-2.

3. RIVUR Trial Investigators, Hoberman A, Greenfield SP, et al. Antimicrobial prophylaxis for children with vesicoureteral reflux. N Engl J Med 2014; 370(25):2367-6.

4. Shipman SA, Helfand M, Moyer VA, Yawn BP. Screening for developmental dysplasia of the hip: a systematic literature review for the US Preventive Services Task Force. Pediatrics 2006; 117(3):e557-76.

5. Rollins MD, Barnhart DC, Greenberg RA, et al. Neurologically intact children with an isolated skull fracture may be safely discharged after brief observation. J Pediatr Surg 2011; 46(7):1342-6.

6. Quinonez RA, Coon ER, Schroeder AR, Moyer VA. When technology creates uncertainty: pulse oximetry and overdiagnosis of hypoxaemia in bronchiolitis. BMJ 2017; 358:j3850.

7. Principi T, Coates AL, Parkin PC, et al. Effect of Oxygen Desaturations on Subsequent Medical Visits in Infants Discharged From the Emergency Department With Bronchiolitis. JAMA Pediatr 2016; 170(6):602-8.

8. Shay DK, Holman RC, Newman RD, et al. Bronchiolitisassociated hospitalizations among US children, 1980-1996. JAMA 1999; 282(15):1440-6.

9. Shay DK, Holman RC, Roosevelt GE, et al. Bronchiolitisassociated mortality and estimates of respiratory syncytial virus-associated deaths among US children, 1979-1997. J Infect Dis 2001; 183(1):16-22.

10. Cunningham S, Rodriguez A, Adams T, et al. Oxygen saturation targets in infants with bronchiolitis (BIDS): a double-blind, randomised, equivalence trial. Lancet 2015; 386(9998):1041-8.

11. Schuh S, Freedman S, Coates A, et al. Effect of oximetry on hospitalization in bronchiolitis: A randomized clinical trial. JAMA 2014; 312(7):712-8.

12. Miglioretti DL, Johnson E, Williams A, et al. The use of computed tomography in pediatrics and associated radiation exposure and estimated cancer risk. JAMA Pediatr 2013; 167(8):700-7.

13. BergmanAB,StammSJ. The morbidity of cardiacnondisease in schoolchildren. N Engl J Med 1967; 276(18):1008-13.

14. Ikeda H, Suzuki N, Takahashi A, et al. Surgical treatment of neuroblastomas in infants under 12 months of age. J Pediatr Surg 1998; 33(8):1246-50.

15. Nuchtern JG, London WB, Barnewolt CE, et al. A prospective study of expectant observation as primary therapy for neuroblastoma in young infants: a Children's Oncology Group study. Ann Surg 2012; 256(4):573-80.

16. Centers for Disease Control and Prevention. Antibiotic resistance threats in the United States 2013. Atlanta, 2013. [Accessed on: June 19, 2018]. Available at: http://www.cdc.gov/drugresistance/ threat-report-2013

17. Quiñonez RA, Schroeder AR. Safely doing less and the new AAP bronchiolitis guideline. Pediatrics 2015; 135(5):793-5.

18. Schroeder AR, Harris SJ, Newman TB. Safely doing less: a missing 
component of the patient safety dialogue. Pediatrics 2011; 128(6):e1596-7.

19. Choosing Wisely ${ }^{\circledR}$ is an initiative of the ABIM Foundation. Philadelphia, PA; ${ }^{\circledR} 2018$ [Accessed on: June 19, 2018]
Available at: www.choosingwisely.org

20. Behrman RE. Some unchanging values of pediatric education during a time of changing technology and practice. Pediatrics 1996; 98(6 Pt 2):1249-54. 